Cahiers $d u$ MONDE RUSSE

\section{Cahiers du monde russe}

Russie - Empire russe - Union soviétique et États indépendants

$51 / 4 \mid 2010$

Sciences humaines et sociales en Russie à l'Âge d'argent

\title{
Karl D. Qualls, From Ruins to Reconstruction
}

\section{Vanessa Voisin}

\section{(2) OpenEdition}

Journals

Édition électronique

URL : https://journals.openedition.org/monderusse/7400

DOI : 10.4000/monderusse. 7400

ISSN : 1777-5388

Éditeur

Éditions de l'EHESS

Édition imprimée

Date de publication : 25 novembre 2010

Pagination : 737-740

ISBN : 978-2-7132-2316-7

ISSN : $1252-6576$

Référence électronique

Vanessa Voisin, "Karl D. Qualls, From Ruins to Reconstruction », Cahiers du monde russe [En ligne], 51/4 | 2010, mis en ligne le 09 décembre 2011, consulté le 03 septembre 2022. URL : http://

journals.openedition.org/monderusse/7400; DOl : https://doi.org/10.4000/monderusse.7400

Ce document a été généré automatiquement le 3 septembre 2022.

Tous droits réservés 


\title{
Karl D. Qualls, From Ruins to Reconstruction
}

\author{
Vanessa Voisin
}

\section{RÉFÉRENCE}

\section{Karl D. QUALLS, From Ruins to Reconstruction. Urban Identity in Soviet Sevastopol}

after World War II. Ithaca - Londres : Cornell University Press, 2009, 214 p.

1 L'étude de Karl D. Qualls sur la reconstruction de Sébastopol après la Seconde Guerre mondiale s'appuie sur sa thèse, qui s'intéressait au processus de « restauration de l'allégeance populaire à travers le plan d'urbanisation » de la ville entre 1944 et 1953. Un chapitre conclusif pousse l'analyse jusqu'à l'époque postsoviétique en révélant la solidité de l'identité urbaine forgée après-guerre. Le livre tire sa valeur de l'originalité du point de vue qui consiste à interroger les grandes questions historiographiques du haut stalinisme à travers l'exemple d'une reconstruction urbaine mêlant enjeux matériels et enjeux symboliques. En effet, le choix de l'échelle microrégionale offre la possibilité de croiser la problématique sociale de la sortie de guerre avec les enjeux politiques, tant locaux que centraux, et enfin avec la dimension identitaire et mémorielle. L'auteur montre à quel point ces aspects sont enchevêtrés.

2 L'approche urbanistique éclaire la montée en puissance des identités locale et nationale et les débats qu'elle a pu susciter à la lumière de l'espace vécu. Se focalisant moins sur les processus mémoriels en tant que tels que sur « le processus de leur création comme outil, ou technique, d'identification des résidents à la cité elle-même et simultanément à la Russie et à l'Union soviétique ", l'auteur insiste sur la complémentarité, plutôt que sur l'opposition, des objectifs des autorités locales et centrales. Ainsi, lorsqu'ils défendaient une approche plus concrète des besoins des citadins, "les fonctionnaires locaux tâchaient de donner corps à la rhétorique stalinienne du souci [de l'État] pour l'individu» (p. 123). Ce constat nuance fortement la vision du haut stalinisme comme triomphe d'une centralisation toute-puissante. Les autorités municipales (soviet, gosplan 
et département de l'architecture) se dressèrent contre la volonté moscovite d'utiliser la reconstruction des villes détruites comme un outil de démonstration de la puissance du grand vainqueur de la guerre, capable, sans aide américaine, de répondre aux besoins de ses citoyens durement éprouvés par le conflit. Les projets urbains, destinés à rompre de façon radicale avec «l'ancien » afin de célébrer la modernité soviétique, cédèrent ainsi place à un urbanisme de proximité qui sut entrecroiser le passé propre de la ville et le discours soviétique sur les « villes héros".

3 S'inscrivant dans la continuité d'A. Weiner, l'auteur montre que ces choix prolongeaient - après la libération - un récit sur la guerre dans le but de maintenir la mobilisation populaire pour les nouveaux sacrifices de la reconstruction. Pourtant, dans cette démarche, les dirigeants locaux rencontrèrent finalement un autre objectif de Moscou : l'intégration de l'identité locale à l'identité nationale. «Comme l'histoire de Sébastopol se concentrait autour de la notion de sacrifice militaire pour la défense de la patrie, la nouvelle vision locale [...] complétait, plutôt qu'elle ne concurrençait, les objectifs soviétiques plus vastes» (p. 10). La situation de la ville en faisait une ville ukrainienne ce qu'elle devint officiellement en 1954 - mais son histoire servait parfaitement le discours russocentré promu par le Kremlin au sortir de la guerre.

4 La principale critique que l'on peut retenir contre ce travail concerne le silence sur certaines sources dont on aurait pu espérer des éclaircissements utiles quant à la formation du «mythe » de Sébastopol. Ainsi, le chapitre I retrace l'apparition de l'idée de « seconde bataille de Sébastopol » (par référence à la guerre de Crimée) à travers la presse d'époque, les écrits du commandant de la défense de la ville en 1942, Oktjabrskij, et les documentaires produits pendant la guerre. Selon l'auteur, les responsables de la reconstruction urbaine après 1945 se fondèrent eux aussi sur l'exaltation de l'identité prérévolutionnaire et militaire de la ville, s'opposant ainsi aux architectes de Moscou qui privilégiaient un discours pansoviétique sur la gloire présente, le rôle de Stalin, et l'aboutissement de l'œuvre accomplie par les pères de la révolution. On ignore cependant si des visions alternatives de la gloire locale furent proposées pendant la même période, dans la presse partisane - fugitivement évoquée - ou dans les nombreux rushes conservés aux archives audiovisuelles de Moscou (RGAKFD). Une analyse génétique du film La Bataille de Sébastopol de Beljaev (1944), seul évoqué, aurait peut-être permis d'aborder les débats suscités en haut lieu par la forme et l'importance de cette mise en rapport des deux conflits. On peut d'ailleurs s'interroger sur la pertinence de ce parallèle dans la mesure où les fictions cinématographiques et les articles de presse exaltant les grandes batailles passées de la Russie contre des envahisseurs furent aussi nombreux que remarquables. De même, la comparaison de La Bataille de Sébastopol avec les autres «Batailles" pour la plupart sorties sur les écrans la même année (La Bataille pour la Biélorussie, La Bataille pour les Pays baltes, La Bataille de Riga) ou avec des films au moins aussi célèbres (les deux films d'Aleksandr Dovženko, La Bataille pour notre Ukraine soviétique de 1943 et La Victoire en Ukraine de 1945) aurait également permis de mieux apprécier la représentativité ou la spécificité du cas de Sébastopol. On ignore enfin à quelle échelle fut diffusé ce discours «localiste» pendant la guerre, puisque l'auteur ne distingue pas systématiquement les articles publiés par la presse locale et par la presse nationale, ni les films destinés aux spectateurs de l'ensemble de l'URSS des films destinés au public local.

5 Le deuxième chapitre, qui traite de la rivalité entre plans d'urbanisme locaux et moscovites, s'appuie sur un corpus de sources plus convaincant. L'analyse des phases de 
cette lutte et des raisons du succès du projet local est intéressante, même si ses responsables ainsi que les circonstances de la décision finale auraient peut-être mérité plus d'éclaircissements. Pour ce qui concerne la position des fonctionnaires locaux de l'appareil d'État, dont l'auteur nous précise l'origine extérieure à la Crimée, leur ralliement à l'exaltation d'une ancienne mémoire locale est attribué à l'expérience partagée des difficultés matérielles de l'après-guerre. Ce vécu quotidien les aurait sensibilisés à la nécessité de penser un plan d'urbanisme respectueux de la mémoire spécifique de la ville. Si l'explication est tout à fait recevable, elle n'exclut pas une intervention des dirigeants locaux du parti, dont l'ouvrage ne souffle mot. Or la finalité propagandiste qui accompagne ces débats, à laquelle l'étude consacre à juste titre une place centrale, relevait bien du département d'agitation et de propagande des comités locaux du parti et, au-dessus, du Comité central. Si ce dernier fut absent des discussions autour du plan de reconstruction urbaine, cela mérite d'être relevé !

Le chapitre III traite des problèmes concrets que l'autorité municipale eut à résoudre dans un contexte général de famine (1946-1947), de pénurie, et d'immigration de maind'œuvre nécessaire à l'industrie comme à la reconstruction. Des exemples très vivants permettent aisément au lecteur de se représenter la gravité de la situation sanitaire de Sébastopol jusqu'en 1953, malgré les efforts créatifs des pouvoirs locaux et des particuliers pour faire face aux besoins. L'action des planificateurs urbains passe toutefois au second plan et leur rôle dans la défense de chantiers essentiels n'est pas très clair. Il semble qu'ils aient surtout fermé les yeux sur les constructions sauvages érigées çà et là, faute d'action centrale.

7 Une analyse comparée, et détaillée, des plans monumentaux des architectes de Moscou et de ceux des architectes locaux, est proposée dans le chapitre suivant. La conclusion confirme la thèse initiale d'une victoire de la symbolique locale et historique sur les projets centraux, orientés vers la célébration de la victoire soviétique. L'auteur relève l'autorité des représentants de la flotte de la mer Noire dans ces débats et la précocité de leurs propres constructions mémorielles.

Un chapitre final montre que cette réinscription de l'histoire immédiate de la ville dans un passé plus lointain et spécifique a permis à l'urbanisme de la Sébastopol d'aprèsguerre de survivre aux soubresauts politiques de la fin du $\mathrm{xx}^{\mathrm{e}}$ siècle. 